\title{
A 68-year-old woman with sleep-onset insomnia
}

\author{
Norman Wolkove MD, Marc Baltzan MD
}

See also review article by McMillan and colleagues on page 1499 and at www.cmaj.ca/lookup/doi/10.1503/cmaj.130025

\begin{abstract}
A 68-year-old woman, who smokes 1 package of cigarettes daily, presents with fatigue, daytime sleepiness and difficulty falling asleep at night. She reports that, for as long as she can remember, her legs "absolutely needed to move" at bedtime. She has not experienced leg pain or cramps but describes a "prickly feeling under the skin." Examinations, including neurologic evaluation, reveal no abnormalities. The results of a complete blood count were within normal limits, and her serum ferritin level was 52 (normal 18-160) $\mu \mathrm{g} / \mathrm{L}$.
\end{abstract}

\section{What is the most likely diagnosis?}

Primary restless legs syndrome is the most likely diagnosis. Restless legs syndrome is a neurologic movement disorder that may adversely affect sleep, and thereby result in daytime fatigue, somnolence and a reduced quality of life. ${ }^{1-4}$ This condition has also been associated with hypertension and cardiovascular disease. ${ }^{4}$ The pathophysiology of restless legs syndrome is incompletely understood. However, dopamine dysfunction in the central nervous system and iron deficiency in the brain have been implicated. ${ }^{1-5}$

Sleep-onset insomnia is typical. Patients describe an overwhelming urge to move, accompanied by unpleasant sensations in their lower limbs. The sensations are often described as "creeping," "burning" or "tingling." These symptoms appear at rest, usually in the evening or at bedtime, and are improved by movement. Patients typically describe voluntarily moving their limbs, stretching, walking or pacing to find relief. Symptoms may also occur during the night, thereby interrupting sleep, and they may occur nightly or on an intermittent basis. ${ }^{1-4}$

Restless legs syndrome is common, affecting $5 \%-15 \%$ of the population, yet it is frequently undiagnosed or mistaken for other conditions. ${ }^{1,3,4}$ Women are affected more often than men, and the prevalence increases with age. ${ }^{1,3,4}$ Over half of patients with primary restless legs syndrome have a family history, and a genetic trait with autosomal dominant transmission has been hypothesized. ${ }^{1,4}$ Restless legs syndrome may also be associated with other conditions, including diabetes, arthritis, Parkinson disease, chronic renal failure, iron deficiency and pregnancy (secondary restless legs syndrome). ${ }^{1,3,4}$ Drugs such as antihistamines, selective serotonin reuptake inhibitors, tricyclic antidepressants and dopamine antagonists can provoke or exacerbate restless legs syndrome. ${ }^{1}$

Other conditions with lower limb symptoms that can be confused with restless legs syndrome include akathisia, leg cramps, peripheral neuropathy, lumbosacral radiculopathy, vascular diseases and the syndrome of painful legs and moving toes. ${ }^{1,3,4}$ Akathisia is characterized by a general restlessness; the sensory complaints of restless legs syndrome are absent, and symptoms usually occur during the day. ${ }^{1,3,4}$ Common leg cramps that occur at night tend to be unilateral, may wake patients from sleep and are described as palpable involuntary muscle contractions. ${ }^{1,3,4}$ Peripheral neuropathy and the sensory-motor changes of lumbosacral disc disease may be distinguished by an abnormal neurologic exam and are not associated with an urge to move..$^{1,3,4}$ Patients with arterial or venous peripheral vascular disease have symptoms that do not have a circadian pattern, and examination may show an absent or diminished pulse, peripheral edema and skin changes. ${ }^{1,3,4}$ The syndrome of painful legs and moving toes is characterized by involuntary flexion and extension of the toes in the context of trauma to the spinal cord, nerve roots or cauda equina. Both the urge to move and a circadian pattern are absent. ${ }^{1,3,4}$

\section{How can the diagnosis of restless legs syndrome be confirmed?}

The diagnosis is based on the typical clinical history. The results of a physical examination are usually normal unless there is an underlying predisposing condition. ${ }^{1,4}$ The National Institutes of
Competing interests: None declared.

Correspondence to: Norman Wolkove, nwolkove@pne.jgh.mcgill .ca

CMAJ 2013. DOI:10.1503 /cmaj.130260 
Health state that the following 4 criteria are essential for diagnosing restless legs syndrome: an urge to move the legs usually accompanied by, or caused by, uncomfortable and unpleasant sensations in the legs; unpleasant sensations that begin or worsen during periods of rest or inactivity; unpleasant sensations that are partially or totally relieved by movement; and unpleasant sensations that are worse in the evening or at night compared with during the day or that only occur in the evening or at night. ${ }^{6}$ Supportive but not essential features include a positive family history, response to dopaminergic treatment, and the presence of periodic limb movements.

\section{Are additional investigations necessary?}

There is no specific laboratory test for restless legs syndrome. However, laboratory tests may reveal secondary causes.

Investigations should include tests for iron levels, because iron deficiency (with or without anemia) is common among those with restless legs syndrome. ${ }^{1-4}$ A sleep study (polysomnography) is not necessary for diagnosis, but it may show periodic limb movements, which may be present in $80 \%-90 \%$ of patients with restless legs syndrome. ${ }^{4}$ Periodic limb movement disorder is characterized by repetitive movements of the extremities (usually the legs), resulting in poor quality of sleep and insomnia. The rhythmic muscular jerks may involve extension of the big toe and can include flexion of the ankle, knee and hip. ${ }^{3,4}$

The principal indications for polysomnography among patients with suspected restless legs syndrome include atypical symptoms, marked daytime somnolence (to rule out other sleep disorders, such as obstructive sleep apnea and periodic limb movements) and if there has been an inadequate response to treatment.?

\section{What are the management options in this patient's case?}

Although not supported by clinical trials, most clinicians recommend avoiding alcohol, caffeine and cigarette smoke. ${ }^{3,4}$ In a randomized controlled study, starting an exercise program was effective in improving the symptoms of restless legs syndrome. ${ }^{8}$

Pharmacologic treatment may not be necessary for those with mild symptoms. Other patients may only require treatment on an intermittent basis, whereas those who have nightly symptoms, such as this patient, usually require long-term therapy.

First-line prescription therapy includes dopaminergic agents (Box 1). ${ }^{9-11}$ Meta-analyses have shown that dopaminergic agents have moderate efficacy in the treatment of restless legs

\begin{tabular}{|ll|}
\hline Box 1: Pharmacologic treatment of restless legs syndrome \\
\hline Drugs
\end{tabular}


syndrome. ${ }^{9-11}$ Opioids, benzodiazepines and antiepileptics are second-line choices. Detailed management guidelines based on systematic reviews and meta-analyses have recently been published by the American Academy of Sleep Medicine. ${ }^{11}$

Iron supplementation has been recommended for patients with a ferritin level of $50 \mu \mathrm{g} / \mathrm{L}$ or lower and may improve symptoms for some patients. ${ }^{1,3}$ However, a recent Cochrane review concluded that there is insufficient evidence to determine if iron therapy is beneficial for the treatment of restless legs syndrome. ${ }^{12}$

\section{The case revisited}

No associated illness was found, and we concluded that the patient had primary restless legs syndrome. She was advised to stop smoking and to begin an exercise program. She was prescribed pramipexole $(0.125 \mathrm{mg} 2-3$ hours before bedtime, later increased to $0.5 \mathrm{mg}$ nightly), which resulted in improved symptoms and sleep.

\section{References}

1. Ekbom K, Ulfberg J. Restless legs syndrome. J Intern Med 2009;266:419-31.

2. National Heart. Lung and Blood Institute working group on restless legs syndrome. Am Fam Physician 2000;62:108-14.

3. Natarajan R. Review of periodic limb movement and restless leg syndrome. J Postgrad Med 2010;56: $157-62$.

4. Bogan RK, Cheray JA. Restless legs syndrome: a review of diagnosis and management in primary care. Postgrad Med 2013;125:99-111.

5. Mizuno S, Mihara T, Miyaoka T, et al. CSF iron, ferritin and transferrin levels in restless legs syndrome. J Sleep Res 2005; 14:43-7.

6. Allen RP, Picchietti D, Henig WA, et al. Restless legs syndrome: diagnostic criteria, special considerations and epidemiology. A report from the Restless Legs Syndrome Diagnosis and Epidemiology Workshop at the National Institute of Health. Sleep Med 2003;4:101-19.

7. Hornyak M, Kotterba S, Trenkwalder C; members of the study group "motor disorders" of the German Sleep Society. Indications for performing polysomnography in the diagnosis and treatment of restless legs syndrome. Somnologie 2001;5:159-62.

8. Aukerman MM, Aukerman D, Bayard M, et al. Exercise and restless legs syndrome: a randomized controlled trial. J Am Board Fam Med 2006;19:487-93.

9. Wilt TJ, MacDonald R, Ouellette J, et al. Pharmacologic therapy for primary restless legs syndrome: a systematic review and meta-analysis. JAMA Intern Med 2013;173:496-505.

10. Scholz H, Trenkwalder C, Kohnen R, et al. Dopamine agonists for restless legs syndrome. Cochrane Database Syst Rev 2011;(3):CD006009.

11. Aurora RN, Kristo DA, Bista SR, et al. The treatment of restless legs syndrome and periodic limb movement disorder in adults — an update for 2012: practice parameters with an evidence-based systematic review and meta-analysis: an American Academy of Sleep Medicine Clinical Practice Guideline. Sleep 2012;35:1039-62.

12. Trotti LM, Bhadriraju S, Becker LA. Iron for restless legs syndrome. Cochrane Database Syst Rev 2012;(5):CD007834.

Affiliations: Department of Medicine (Wolkove); and Department of Epidemiology, Biostatistics and Occupational Health (Baltzan), Mount Sinai Hospital, McGill University, Montréal, Que.

Contributors: Both authors contributed to the conception and design and to the writing and revising of the manuscript. Both authors approved the final version submitted for publication. 